2007-0381

45th AIAA Aerospace Science Meeting and Exhibit, 8-11 January 2007, Reno, Nevada

\title{
Verified Computations of Laminar Premixed Flames
}

\author{
Ashraf N. Al-Khateeb, Joseph M. Powers $\dagger^{\dagger}$ and Samuel Paolucci ${ }^{\ddagger}$ \\ University of Notre Dame, Notre Dame, Indiana, 46556-5637, USA
}

\begin{abstract}
The required spatial discretization to capture all detailed continuum physics in the reaction zone for one-dimensional steady laminar premixed hydrogen-air flames described by detailed kinetics and multi-component transport is accurately estimated a priori by a simple mean free path calculation. To verify this, a robust method has been developed to rigorously calculate the finest length scale a posteriori. The method reveals that the finest length scale is at the micron-level. This result is consistent with an estimate from the underlying molecular collision theory, and orders of magnitude smaller than the discretization scales employed in nearly all multi-dimensional and/or unsteady laminar premixed flame simulations in the literature.
\end{abstract}

\section{Introduction}

It is well understood that in any mathematically based scientific theory, associated computations should have fidelity with the underlying mathematics, and the underlying mathematical model has to represent the observed physics. The first issue is demonstrated by comparing computational results with another known solution and/or performing a formal grid convergence study, while the second issue is demonstrated by comparing the computational predictions with experimental data. Addressing these two issues, in this order, is a necessity in any computational study to build confidence in both the simulation strategy and the underlying mathematical model.

The exercise of demonstrating the harmony of the discrete solution with the foundational mathematics is known as verification. ${ }^{1}$ For multi-scale problems, verification is difficult due to the range of the spatiotemporal scales, which may span many orders of magnitude. In this kind of problem, usually modeled by highly nonlinear equations, significant coupling across the scales can occur, so that errors at small scales can rapidly cascade to the large scales. Moreover, the strength of the coupling across the scales is not known a priori. So, all the physical scales of the mathematical model, temporal and spatial, have to be captured in order to have full confidence that predictions are repeatable, grid-independent, and thus verifiable. Subsequently, in the validation step one can choose what physical phenomena and to what accuracy one wants to reproduce experiments.

The main aim of this paper is to rigorously determine the required spatial resolution to capture all physical scales in a standard multi-scale problem: the steady one-dimensional laminar premixed flame propagating freely at atmospheric pressure in a stoichiometric mixture of hydrogen-air described by detailed kinetics and multi-component transport. Here, the robust method to calculate the length scales employed in Powers and Paolucci ${ }^{2,3}$ for gas phase detonation is implemented with modification for deflagration. The method is robust in that it has little dependence on the details of the underlying numerical method used to calculate the laminar flame. It simply requires a local determination of the state of the system, which is followed by a Jacobian formulation, and a generalized eigenvalue analysis. As such, it is able to estimate with great accuracy the length scales on a fundamental mathematical, non-numerical, basis. The minimum length scale which must be resolved in order for the mathematical model to be verified is thus determined.

In the first section, the governing partial differential equations (PDEs) for unsteady reactive flow are presented. This is followed by a reduction of the PDEs into a system of differential algebraic equations (DAEs)

*Ph.D. Candidate, Department of Aerospace and Mechanical Engineering, AIAA Student Member, aalkhate@nd.edu.

${ }^{\dagger}$ Associate Professor, Department of Aerospace and Mechanical Engineering, AIAA Associate Fellow, powers@nd.edu.

${ }^{\ddagger}$ Professor, Department of Aerospace and Mechanical Engineering, AIAA Member, paolucci@nd.edu.

Copyright (C) 2007 by Joseph M. Powers. Published by the American Institute of Aeronautics and Astronautics, Inc. with permission. 
which describes the spatial evolution of the state variables. Following a short description of the generalized eigenvalue analysis and length scale determination, the standard form of the equations is delineated, and a brief description of the numerical method is presented. Next, the numerical algorithm is verified against calculations given by Smooke et al. ${ }^{4}$ Then, the mathematical model is validated against experimental data compiled by Dixon-Lewis. ${ }^{5}$

For the main results of the study, it is desirable to have a physical solution in all regions of the laminar flame. So, in order to suppress numerical anomalies near the cold boundary so as to fully expose the behavior in all regions of the flame, the initial mixture temperature is raised to $800 \mathrm{~K}$. As a result, a fully resolved prediction of a laminar premixed flame in a stoichiometric hydrogen-air mixture initially at atmospheric pressure is achieved. Then, all the length scales over which the system evolves are shown, and the finest length scale is compared to predictions of a simple molecular theory. Moreover, this comparison is presented for a wide range of pressures. Finally, a comparison between the grid resolution utilized in more general recent studies with the required length scale to resolve the underlying one-dimensional steady laminar flame structure, predicted by the eigenvalue analysis, is given before specific conclusions are stated.

\section{Mathematical Model}

\section{II.A. Governing Equations}

The following unsteady equations ${ }^{6}$ describe the system under consideration, a one-dimensional adiabatic laminar premixed mixture of $N$ molecular species composed of $L$ atomic elements which undergo $J$ reversible reactions with no body force present:

$$
\begin{aligned}
\frac{\partial \rho}{\partial \tilde{t}} & =-\frac{\partial}{\partial \tilde{x}}(\rho \tilde{u}), \\
\frac{\partial}{\partial \tilde{t}}(\rho \tilde{u}) & =-\frac{\partial}{\partial \tilde{x}}\left(\rho \tilde{u}^{2}+p-\tau\right), \\
\frac{\partial}{\partial \tilde{t}}\left(\rho\left(e+\frac{\tilde{u}^{2}}{2}\right)\right) & =-\frac{\partial}{\partial \tilde{x}}\left(\rho \tilde{u}\left(e+\frac{\tilde{u}^{2}}{2}+\frac{p}{\rho}-\frac{\tau}{\rho}\right)+J^{q}\right), \\
\frac{\partial}{\partial \tilde{t}}\left(\rho Y_{i}\right) & =-\frac{\partial}{\partial \tilde{x}}\left(\rho \tilde{u} Y_{i}+J_{i}^{m}\right)+\dot{\omega}_{i} M_{i}, \quad i=1, \ldots, N-1 .
\end{aligned}
$$

The independent variables are the spatial coordinate $\tilde{x}$ and the time $\tilde{t}$. The dependent variables are mixture density $\rho$, mixture velocity $\tilde{u}$, pressure $p$, viscous stress $\tau$, mass-based specific internal energy of the mixture $e$, total heat flux $J^{q}$, and for the $i^{t h}$ specie, $Y_{i}, J_{i}^{m}$, and $\dot{\omega}_{i}$, which are the the mass fraction, the diffusive mass flux, and the molar production rate per unit volume, respectively. The parameter $M_{i}$ is the molecular mass of specie $i$. Equations (1-3) describe the conservation of mass, linear momentum, and energy, respectively. Equation (4) is an evolution equation for $N-1$ species.

For this system, the constitutive relations for diffusive mass fluxes and heat flux are

$$
\begin{aligned}
J_{i}^{m} & =\rho \sum_{\substack{k=1 \\
k \neq i}}^{N} \frac{M_{i} D_{i k} Y_{k}}{M}\left(\frac{1}{\chi_{k}} \frac{\partial \chi_{k}}{\partial \tilde{x}}+\left(1-\frac{M_{k}}{M}\right) \frac{1}{p} \frac{\partial p}{\partial \tilde{x}}\right)-D_{i}^{T} \frac{1}{T} \frac{\partial T}{\partial \tilde{x}}, \quad i=1, \ldots, N-1, \\
J^{q} & =q+\sum_{i=1}^{N} J_{i}^{m} h_{i}-\Re T \sum_{i=1}^{N} \frac{D_{i}^{T}}{M_{i}}\left(\frac{1}{\chi_{i}} \frac{\partial \chi_{i}}{\partial \tilde{x}}+\left(1-\frac{M_{i}}{M}\right) \frac{1}{p} \frac{\partial p}{\partial \tilde{x}}\right),
\end{aligned}
$$

where,

$$
\begin{aligned}
q & =-k \frac{\partial T}{\partial \tilde{x}} \\
p & =\Re T \sum_{i=1}^{N} \frac{\rho Y_{i}}{M_{i}}
\end{aligned}
$$

In Eqs. (5-8), the new dependent variables are mixture-average molecular mass $M$, temperature $T$, Fourier's heat flux $q$, and for the $i^{t h}$ specie, $\chi_{i}$ and $h_{i}$, which are the mole fraction and the mass-based spe- 
cific enthalpy. The variables $D_{i k}, k$, and $D_{i}^{T}$ are the multi-component diffusion coefficients, the temperaturedependent mixture thermal conductivity, and the thermal diffusion coefficient of specie $i$. A constant parameter is the universal gas constant $\Re=8.314 \times 10^{7} \mathrm{erg} / \mathrm{mole} / \mathrm{K}$. Equations (5-6) are appropriate for a mixture of ideal gases, ${ }^{7}$ and describe multi-component mass diffusion fluxes including the Soret effect, and the heat flux including the Dufour effect. Equation (7) defines Fourier's law. Equation (8) is the thermal state equation for an ideal gas mixture. This system of equations is completed by adopting an appropriate set of additional constitutive relations, (e.g. the law of mass action, the Arrhenius reaction rate, the temperature-dependent enthalpy, and entropy). Full details are given by Al-Khateeb et al. ${ }^{8}$ and Singh et $a l .{ }^{9}$

The complete system is simplified by reducing it into a system of ordinary differential equations (ODEs). The time-dependent behavior of the system is relaxed to a steadily propagating flame front with constant, albeit unknown, flame speed $S$. The low-Mach number assumption is adopted. This assumption is reasonable for deflagration, ${ }^{10}$ and implies that for a fixed mass flux the momentum equation no longer need be considered. A systematic reduction of the complete system is given in Ref. 8.

As a result, the governing equations are recast in the form

$$
\begin{aligned}
\frac{d}{d x}(\rho u) & =0, \\
\frac{d}{d x}\left(\rho u h+J^{q}\right) & =0, \\
\frac{d}{d x}\left(\rho u Y_{l}^{e}+J_{l}^{e}\right) & =0, \quad l=1, \ldots, L-1, \\
\frac{d}{d x}\left(\rho u Y_{i}+J_{i}^{m}\right) & =\dot{\omega}_{i} M_{i}, \quad i=1, \ldots, N-L .
\end{aligned}
$$

The spatial coordinate $x$ is a flame front-attached coordinate, $u$ is the mixture velocity in the flame frame, $h$ is the mass-based specific enthalpy of the mixture, $Y_{l}^{e}$ are the element mass fractions, and $J_{l}^{e}$ are the element mass fluxes. For Eqs. (9-12), that describe the steadily propagating laminar premixed flame, the appropriate set of boundary conditions is

$$
\begin{aligned}
& x=0: \quad T=T_{o}, \quad Y_{i}+\frac{J_{i}^{m}}{\rho_{o} S}=Y_{i o}, \quad i=1, \ldots, N-1, \\
& x \rightarrow \infty: \quad \frac{d T}{d x} \rightarrow 0, \quad \frac{d Y_{i}}{d x} \rightarrow 0, \quad i=1, \ldots, N-1, \\
& x=x_{f}: \quad T=T_{f},
\end{aligned}
$$

where $x_{f}$ is a specified spatial point and $T_{f}$ is the specified temperature at that location. ${ }^{11}$ These are commonly used to study deflagration, though other formulations are possible.

At this stage the variable $S$ is considered a fixed parameter for a given calculation; an iterative technique is used to determine $S$ so that all boundary conditions are satisfied. The equations are most conveniently posed as a set of $2 N+2$ DAEs in terms of $2 N+2$ state variables; species mass fraction $Y_{i},(i=1, \ldots, N)$, species mass flux $J_{i}^{m},(i=1, \ldots, N)$, temperature $T$, and Fourier's heat flux $q$. This system, in a compact representation, is

$$
\mathbf{A} \cdot \frac{d \mathbf{z}}{d x}=\mathbf{f}
$$




$$
\mathbf{A}=\left[\begin{array}{ccc}
\mathcal{D} & \mathbf{0} & \mathbf{0} \\
\dot{\mathcal{M}} & \mathcal{I} & \mathbf{0} \\
\mathbf{0} & \mathbf{0} & \mathcal{Q}
\end{array}\right], \mathbf{z}=\left[\begin{array}{c}
Y_{1} \\
\vdots \\
Y_{N} \\
J_{1}^{m} \\
\vdots \\
J_{N}^{m} \\
T \\
q
\end{array}\right], \mathbf{f}=\left[\begin{array}{c}
\frac{J_{1}^{m} M}{\rho M_{1}} \\
\vdots \\
\frac{J_{N}^{m} M}{\rho M_{N}} \\
\dot{\omega}_{1} M_{1} \\
\vdots \\
\dot{\omega}_{N-L} M_{N-L} \\
\rho u Y_{1}^{e}+J_{1}^{e}-\left(\rho_{o} S Y_{1 o}^{e}\right) \\
\vdots \\
\rho u Y_{L}^{e}+J_{L}^{e}-\left(\rho_{o} S Y_{L o}^{e}\right) \\
\rho u h+J^{q}-\left(\rho_{o} S h_{o}\right) \\
q
\end{array}\right]
$$

where,

$$
\begin{aligned}
\mathcal{D} & =D_{i k}-\sum_{\substack{m=1 \\
m \neq i}}^{N} \frac{D_{i m} Y_{m} M}{M_{k}}, \quad i=1, \ldots, N, \quad k=1, \ldots, N, \\
\dot{\mathcal{M}} & =\left[\begin{array}{c:c}
\rho_{O} S \mathbf{I}_{(N-L) \times(N-L)} & \mathbf{0}_{(N-L) \times L} \\
\hdashline \mathbf{0}_{L \times(N-L)} & \mathbf{0}_{L \times L}
\end{array}\right], \\
\mathcal{I} & =\left[\begin{array}{c:c}
\mathbf{I}_{(N-L) \times(N-L)}-\mathbf{0}_{(N-L) \times L}- \\
\hdashline \mathbf{0}_{L \times(N-L)} & \mathbf{0}_{L \times L}
\end{array}\right], \\
\mathcal{Q} & =\left[\begin{array}{cc}
0 & 0 \\
-k & 0
\end{array}\right] .
\end{aligned}
$$

The dynamical system, Eq. (16), and the boundary conditions, Eqs. (13-15), are useful for length scale analysis. Direct solution of this system for the reaction zone structure is possible, in principle. However, the problem can be shown to be a high order shooting problem rendering direct solution difficult.

\section{II.B. A Posteriori Length Scale Analysis}

To accurately determine the length scales over which the system evolves, an eigenvalue analysis can be applied to Eq. (16). Since $\mathbf{A}$ is singular, the standard eigenvalue analysis is not applicable. Instead, the generalized eigenvalues of this dynamical system can be calculated. ${ }^{12}$

Assume first that $\mathbf{z}=\widehat{\mathbf{z}}(x)$ has been determined by some appropriate numerical method so that $\widehat{\mathbf{z}}(x)$ satisfies Eqs. $(13-15,16)$. Consider then an arbitrary spatial point $x=x^{*}$ at which the state variables are $\mathbf{z}=\widehat{\mathbf{z}}\left(x^{*}\right)=\mathbf{z}^{*}$. By defining the perturbation from $\widehat{\mathbf{z}}(x)$ as $\tilde{\mathbf{z}}(x)=\mathbf{z}(x)-\widehat{\mathbf{z}}(x)$, linearizing Eq. (16) about $x=x^{*}$, and adopting the standard assumption that

$$
\tilde{\mathbf{z}}=\mathbf{v} e^{\lambda x},
$$

where $\lambda$ and $\mathbf{v}$ are constants yield the generalized eigenvalue problem

$$
\lambda \mathbf{A}^{*} \cdot \mathbf{v}=\mathbf{B}^{*} \cdot \mathbf{v},
$$

where $\lambda$ is in general a complex number denoting the generalized eigenvalue, $\mathbf{v}$ is the corresponding generalized eigenvector, and $\mathbf{A}^{*}$ and $\mathbf{B}^{*}$ are matrices of size $(2 N+2) \times(2 N+2)$. Full details are given in Ref. 8 . Solving for $\lambda_{i}, i=1, \ldots, 2 N-L$, then using Eq. (22), it is easily seen that the length scales over which the dependent variables evolve are given by the reciprocal of the real part of these eigenvalues,

$$
\ell_{i}=\frac{1}{\left|\operatorname{Re}\left(\lambda_{i}\right)\right|}, \quad i=1, \ldots, 2 N-L .
$$

By evaluating the eigenvalues at each spatial point, the length scales over which the system evolves through the reaction zone are determined. As a result, the minimum size of discretization to capture the 
finest scale of the system can be determined. In general, the eigenvalues are complex, where the reciprocals of the real parts provide the length scales of amplitude growth, and the reciprocals of the imaginary parts represent the oscillatory length scale. In this work, the eigenvalues are purely real, except for some limited regions.

\section{II.C. Standard Form of the Equations}

The less refined, but more compact, form which commonly appears in the literature to model stationary laminar premixed flames, $c f$. Refs. 11,13,14, are obtained from Eqs. (1-4) by following the same approach as Aris $^{15}$ to arrive at

$$
\begin{aligned}
\frac{d}{d x}(\rho u) & =0, \\
\rho u c_{p} \frac{d T}{d x}+\frac{d q}{d x}+\sum_{i=1}^{N}\left(J_{i}^{m} \frac{d h_{i}}{d x}+\dot{\omega}_{i} M_{i} h_{i}\right) & =0, \\
\rho u \frac{d Y_{i}}{d x}+\frac{d J_{i}^{m}}{d x} & =\dot{\omega}_{i} M_{i}, \quad i=1, \ldots, N-1 .
\end{aligned}
$$

A solution for this boundary value problem, Eqs. (25-27) with the boundary conditions Eqs. (13-15), can be obtained by discretizing the spatial domain using finite differences, and the resulting algebraic system of equations are solved iteratively using a damped modified Newton's method, where the solution iterate is brought into the convergence domain by using pseudo-time integration. ${ }^{16}$

\section{Computational Method}

A double precision FORTRAN-77 code has been developed and linked with the International Mathematical and Statistical Libraries (IMSL) routines DFDJAC for Jacobian evaluation, DEVLRG for eigenvalues estimation, DGVLRG for generalized eigenvalues estimation, and a double precision version of the public domain edition of the CHEMKIN package ${ }^{17,18}$ to obtain kinetic rates and thermodynamics properties, a double precision version of the public domain edition of the TRANSPORT package ${ }^{19}$ to calculate multi-component transport properties of species, and a double precision version of the public domain edition of the PREMIX algorithm ${ }^{16}$ to obtain the steady structure of adiabatic laminar premixed flames.

In this study, the resolved structure is obtained by solving the standard form, Eqs. (25-27), and the eigenvalues are calculated by using the dynamical system form, Eq. (16). Moreover, all results are obtained on a grid that has been adaptively refined to capture regions of steep gradient. A second order central difference scheme has been employed to discretize all the spatial derivatives. The mass and heat fluxes have been estimated at intermediate grid points to maintain second order accuracy. Moreover, all the calculations presented in this work were performed on a single processor $3.2 \mathrm{GHz}$ Hewlett-Packard machine, and typical calculations for the eigenvalues were completed within one minute.

\section{Results}

A stoichiometric hydrogen-air mixture at $p_{o}=1 \mathrm{~atm}$ has been considered, where the initial molar ratio is given by $2 \mathrm{H}_{2}+\mathrm{O}_{2}+3.76 \mathrm{~N}_{2}$. A kinetic model identical to that of Smooke et al. ${ }^{4}$ with $L=3$ elements, $N=9$ species, and $J=19$ reversible reactions is used. In this mechanism, the reactant species are $\mathrm{H}_{2}, \mathrm{O}_{2}, \mathrm{H}, \mathrm{O}, \mathrm{OH}, \mathrm{HO}_{2}, \mathrm{H}_{2} \mathrm{O}_{2}$, and $\mathrm{H}_{2} \mathrm{O}$. The inert diluent for the mixture is $\mathrm{N}_{2}$.

\section{IV.A. Mathematical Verification and Experimental Validation}

A necessary verification is to achieve the same results that have been obtained in previous studies. To this end, a calculation is performed to reproduce the temperature and species profiles of a stoichiometric, atmospheric pressure hydrogen-air flame found in Smooke et al. ${ }^{4}$ Equations (25-27) with the boundary conditions Eqs. (13-15) are solved, where this mathematical model is identical to the one described in Ref. 4. The specified spatial point is $x_{f}=0.05 \mathrm{~cm}$, the specified temperature is $T_{f}=400 \mathrm{~K}$, and the temperature of the unburned mixture is $T_{o}=298 \mathrm{~K}$. In this particular calculation, the Dufour effect in the heat flux model is neglected, while the Soret effect in the mass flux model is considered to match the model in Ref. 4. 
Although considering one of these terms and neglecting the other violates Onsager reciprocity, this is done here for verification purposes only. The results are illustrated in Fig. 1; it is easily checked that the stationary flame structure is identical to that of Ref. 4.

Next, a comparison with experimental results addresses the question as to whether the model is a good representation of the observable physics. For validation purposes, a series of calculations is performed on an atmospheric pressure hydrogen-air laminar premixed flame initially at $T_{o}=298 \mathrm{~K}$. At different $H_{2}$ percentages in the mixture, the flame speed is determined. A comparison between the calculated flame speeds and the data compiled by Dixon-Lewis ${ }^{20}$ is presented in Fig. 2. It is clear that the computational predictions lie within the scatter of the experimental data, and they are as good as have found by others. ${ }^{4,20}$

\section{IV.B. Stoichiometric Hydrogen-Air Premixed Laminar Flame}

Next, the PREMIX code ${ }^{16}$ is used for determining the stationary structure of a one-dimensional, stoichiometric, adiabatic, $2 \mathrm{H}_{2}+\mathrm{O}_{2}+3.76 \mathrm{~N}_{2}$ premixed laminar flame at atmospheric pressure. The specified temperature is $T_{f}=900 \mathrm{~K}$, the specified temperature location is assigned at $x_{f}=2.30 \mathrm{~cm}$, and the mixture temperature at the cold boundary is $T_{o}=800 \mathrm{~K}$.

Fully resolved steady temperature and species profiles for $T_{o}=800 \mathrm{~K}$ are shown in Figs. 3 and 4. Although linear scales are usually used in the literature, here log-log and semi-log scales have been employed to better illustrate the disparate scales. Figure 3 shows the spatial distribution of species mass fractions throughout the entire flame zone. At $x \approx 10^{-4} \mathrm{~cm}$, the minor species growth rates change slightly, which reveals that significant dissociation reactions at this scale are induced. Another increase in the minor species mass fraction growth rates is noted at $x \approx 10^{-2} \mathrm{~cm}$, which indicates the occurrence of more vigorous chemical interaction of the minor species. For $10^{-2}<x<2.30 \times 10^{0} \mathrm{~cm}$, the minor species mass fractions continue to increase rapidly with different growth rates. On the other hand, the major species $\mathrm{H}_{2}, \mathrm{O}_{2}$, and $\mathrm{N}_{2}$ have essentially constant mass fractions. Just past $x=2.20 \times 10^{0} \mathrm{~cm}$, which is near the end of the preheat zone, all the species mass fractions undergo significant change, and the radicals' mass fractions reach their maximum values. At $x=2.40 \times 10^{0} \mathrm{~cm}$, exothermic recombination of radicals commences forming the predominant product $\mathrm{H}_{2} \mathrm{O}$. This zone extends up to $x=1.39 \times 10^{1} \mathrm{~cm}$; after that, the system comes to an equilibrium where all the spatial gradients vanish. To confirm this, the spatial domain was extended to $x=1.00 \times 10^{2} \mathrm{~cm}$, but no further changes were noted.

In Fig. 4, the temperature profile is presented. At $x \approx 2.20 \times 10^{0} \mathrm{~cm}$, the reaction undergoes a particularly vigorous stage in which the change in the temperature is significant. Thus, the ignition point can be assigned; it is defined as the point where the temperature gradient $d T / d x$ reaches a maximum value. Also, this particular point defines the end of the preheat zone and the beginning of the reaction zone, which extends to the equilibrium. For this case the ignition point is assigned at $x=2.315 \times 10^{0} \mathrm{~cm}$, and the reaction zone length, defined as the distance from the ignition point up to the location where the temperature reaches 0.99 of its equilibrium value, is $\ell_{\text {reaction }}=1.16 \times 10^{1} \mathrm{~cm}$.

Having the fully resolved structure in hand, the local Jacobian and the spatial generalized eigenvalues are calculated from the cold boundary to near equilibrium. As a result, the local length scales $\ell_{i}$ are predicted throughout the domain, Fig. 5. The multi-scale nature of the problem and the length scales over which the species evolve are clearly shown. The finest length scale and the largest length scale for this system vary from $7.60 \times 10^{-4} \mathrm{~cm}$ and $1.62 \times 10^{7} \mathrm{~cm}$ in the preheat zone to $2.41 \times 10^{-4} \mathrm{~cm}$ and $2.62 \times 10^{0} \mathrm{~cm}$ in the reaction zone, respectively.

In addition to the values reported in Fig. 5, the eigenvalues were checked by calculation with other standard algorithms. All algorithms returned equivalent eigenvalues corresponding to fine length scales. However, numerical errors induced some discrepancies in the less important coarse length scale estimates. The least numerical error in the physical eigenvalues was noted in the direct calculation of the eigenvalues; however, this algorithm returned $L=3$ spurious eigenvalues, which were overruled by the more robust generalized eigenvalue method.

The evolution of a particular species is not associated with a particular length scale, since the species mass fractions depend on local linear combinations of all eigenmodes. So, the species mass fractions vary on these scales through the entire domain. The important finest scale is $\ell_{\text {finest }}=2.41 \times 10^{-4} \mathrm{~cm}$, which occurs at equilibrium. The predicted finest length scale and the smallest scale over which the species vary, $x=10^{-4} \mathrm{~cm}$, are nearly identical. Moreover, the finest length scale effect in the preheat zone can be observed in the variation of the minor species mass fractions, which ensures the consistency between the eigenvalue-determined finest length scale and the smallest scale over which the species vary. As the system 
approaches equilibrium, all of the eigenvalues are real: half are positive, and half are negative. Thus, the equilibrium point is a high order saddle node.

\section{IV.C. Mean Free Path Estimate}

Remarkably, it is possible to develop a simple a priori estimate for $\ell_{\text {finest }}$ predicted a posteriori by the calculations of the previous section. Such an estimate is useful in providing a lower bound for computational grid resolution necessary to guarantee fully resolved continuum calculations. The finest length scale can be shown to be close to the mixture mean free path scale $\ell_{m f p}$, which is the cutoff length scale associated with continuum theories. Physically meaningful results are available at or above the mean free path. A simple estimate for the mean free path is given by Vincenti and Kruger, ${ }^{21}$

$$
\ell_{m f p}=\frac{M}{\sqrt{2} \mathcal{N} \pi d^{2} \rho} .
$$

Here, the new parameters are $d$ the molecular collision cross-section diameter and $\mathcal{N}=6.0225 \times$ $10^{-23}$ mole $e^{-1}$ Avogadro's number. For the calculation of $\ell_{m f p}$, the estimate of $d=3.70 \times 10^{-8} \mathrm{~cm}$ for air is adopted from Ref. 21; $M=28.00 \mathrm{~g} /$ mole and $\rho=1.11557 \times 10^{-4} \mathrm{~g} / \mathrm{cm}^{3}$ were easily quantified for the mixture from the PREMIX calculations. The estimate reveals that $\ell_{m f p}=5.87 \times 10^{-5} \mathrm{~cm}$, which is roughly one order of magnitude smaller than the continuum-based $\ell_{\text {finest }}$.

Following the same procedure, a comparison between the predicted finest length scale $\ell_{\text {finest }}$, the mean free path length scale $\ell_{m f p}$, and the reaction zone length $\ell_{\text {reaction }}$ over a wide range of pressures is presented in Fig. 6. It reveals that the estimated finest length scale is well correlated with the mean free path and that both $\ell_{m f p}$ and $\ell_{\text {finest }}$ decrease similarly as pressure is increased. This is consistent with the fact that the parameters used in the constitutive models for the continuum theory have as their foundation the averaged nature of the more fundamental collision theory. In addition, both $\ell_{m f p}$ and $\ell_{\text {finest }}$ are at least three orders of magnitude less than the reaction zone length $\ell_{\text {reaction }}$.

This approach has been extended to several hydrocarbon mixtures (methane, ethane, propane, ethylene, and acetylene), a wide range of fuel-air ratios, and another reactive flow case, the Chapman-Jouguet detonation. Several detailed kinetics models have been adopted (e.g. GRI-3.0, GRI-1.2). The results of these extensions are given in detail in Ref. 8. It was found that the a priori mean free path estimate predicted the finest length scale accurately for all cases.

\section{IV.D. Comparison with Previously Published Results}

Though this work focuses on estimating the length scales of one-dimensional steady laminar premixed flames, the estimates provide bounds for other problems where multi-dimensional and unsteady effects are simulated. In this section, a comparison between the predicted finest length scale and the utilized discretization in some of the best calculations of laminar premixed flames in hydrogen-air mixtures is presented. The results of these calculations are summarized in Table 1, which is organized such that for each study the initial mixture molar ratio, temperature, pressure, reaction zone length $\ell_{\text {reaction }}$, estimated mean free path length $\ell_{m f p}$, finest length scale $\ell_{\text {finest }}$ predicted by the generalized eigenvalue analysis, and grid resolution $\Delta x$ employed are listed, respectively. In all cases, the predicted finest length scales are at the micron-level, and they are well correlated with the associated cutoff length scales admitted by the continuum theory.

Katta and Roquemore ${ }^{22}$ investigated the structure of an axi-symmetric premixed hydrogen-air jet flame using a time-dependent two-dimensional algorithm. The utilized discretization was nonuniform, and the minimum grid size in the axial direction was $2.50 \times 10^{-2} \mathrm{~cm}$. The detailed kinetics model consisted of $N=11$ species and $J=20$ reversible reactions. It has a typographical error in reaction $5-6$ which is unbalanced. This error is corrected by returning to the work of Westbrook ${ }^{23}$ and adopting the corresponding reactions.

Thiele et al. ${ }^{24}$ used a time-dependent two-dimensional model to simulate the spark-ignition in a quiescent hydrogen-air mixture described by a detailed kinetics. The reaction mechanism consists of $N=9$ species and $J=38$ irreversible reactions, adopted from the work of Warnatz et al. ${ }^{25}$ Although the grid discretization in this study is not mentioned, the predicted required length scale, $7.56 \times 10^{-4} \mathrm{~cm}$, to fully resolve such a system was beyond the existing computational capabilities.

Patnaik and Kailasanath ${ }^{26}$ used a detailed kinetics model extracted from the work of Burks and Oran ${ }^{27}$ to simulate a two-dimensional burner-stabilized hydrogen-air flame. The extracted model consists of $J=48$ elementary reactions involving $N=9$ species, but the original model has typographical errors in reactions 
4 and 12, which are unbalanced. These errors are corrected by returning to the work of Baulch et al. ${ }^{28}$ and Hampson et al. ${ }^{29}$ and adopting the corresponding reactions. The spatial resolution in this study was nonuniform, though the average grid size was $\Delta x=3.54 \times 10^{-2} \mathrm{~cm}$.

The main result from Table 1 is that none of these studies have utilized a grid resolution $\Delta x$ that is less than or equal to the finest length scale $\ell_{\text {finest }}$ which is required to have unambiguously resolved results for a steady one-dimensional laminar premixed flame in comparable mixture under the same conditions. Moreover, the utilized grid resolution $\Delta x$ is at least two orders of magnitude greater than $\ell_{\text {finest }}$. In each study, different physical phenomena are simulated, and the mathematical models that are used vary, but the commonality in all studies is the usage of a detailed kinetics model to simulates flame in a premixed hydrogen-air mixture.

Lastly, our results are in rough agreement with independent estimates found in direct numerical simulation (DNS) of turbulent reacting flows. In a recent study, Chen et al., ${ }^{30}$ present a two-dimensional DNS of autoignition at constant volume and high pressure of hydrogen-air described by detailed kinetics. The domain size was $4.1 \mathrm{~mm} \times 4.1 \mathrm{~mm}$, and the calculations required a grid resolution of $\Delta x=4.30 \times 10^{-4} \mathrm{~cm}$ to resolve the ignition fronts, which is similar in magnitude to the finest length scale predicted here.

\section{Conclusion}

The present one-dimensional steady calculations reveal that for an adiabatic laminar premixed flame freely propagating in stoichiometric mixtures of hydrogen-air described by detailed kinetics and multi-component transport, the required grid resolution to formally resolve the modeled flow structures is at the micron-level. This length scale has been predicted by utilizing a rigorous eigenvalue analysis. The length scale predictions are fully reflective of the underlying physics and not the particular numerical method chosen. This has been verified by showing that the finest length scale is well-correlated with the mean free path cutoff length scale estimated from kinetic theory. Thus, it is possible to use a simple mean free path calculation as an a priori estimate of the lower bound for grid discretization. Related calculations of unsteady and multi-dimensional laminar flames in the literature typically employ much larger discretizations than suggested by the present analysis. The full consequences of this under-resolution await rigorous linear and non-linear stability analysis as well as DNS in order to be determined.

\section{Acknowledgments}

The authors recognize the support of the Chemistry Division of Argonne National Laboratory and helpful conversations with Dr. Michael J. Davis of Argonne. The first author acknowledges the partial support of this work from the Center for Applied Mathematics at University of Notre Dame.

\section{References}

\footnotetext{
${ }^{1}$ Oberkampf, W. L., and Trucano, T. G., "Verification and Validation in Computational Fluid Dynamics," Progress in Aerospace Science, Vol. 38, No. 3, 2002, pp. 209-272.

${ }^{2}$ Powers, J. M., and Paolucci, S., "Accurate Spatial Resolution Estimates for Reactive Supersonic Flow with Detailed Chemistry," AIAA Journal, Vol. 43, No. 5, 2005, pp. 1088-1099.

${ }^{3}$ Powers, J. M., and Paolucci, S., "Accurate Estimates of Fine Scale Reaction Zone Thicknesses in Hydrocarbon Detonations," AIAA 2006-0950, 2006.

${ }^{4}$ Smooke, M. D., Miller, J. A., and Kee, R. J., "Determination of Adiabatic Flame Speeds by Boundary Value Methods," Combustion Science and Technology, Vol. 34, Nos. 1-6, 1983, pp. 79-90.

${ }^{5}$ Dixon-Lewis, G., "Flame Structure and Flame Reaction Kinetics. I. Solution of Conservation Equations and Application to Rich Hydrogen-Oxygen Flames," Proceedings of the Royal Society of London. Series A, Vol. 298, No. 1455, 1967, pp. $495-513$.

${ }^{6}$ Bird, R. B., Stewart, W. E., and Lightfoot, E. N., Transport Phenomena, John Wiley, New York, 1960, pp. 554-591.

${ }^{7}$ Merk, H. J., "The Macroscopic Equations for Simultaneous Heat and Mass Transfer in Isotropic, Continuous and Closed Systems," Applied Scientific Research, A, Vol. 8, No. 1, 1959, pp. 73-99.

${ }^{8}$ Al-Khateeb, A. N., Powers, J. M., and Paolucci, S., "Length Scale Issues in Combustion Modelling," Combustion Theory and Modelling, submitted for publication.

${ }^{9}$ Singh, S., Rastigejev, Y., Paolucci, S., and Powers, J. M., "Viscous Detonation in $\mathrm{H}_{2}-\mathrm{O}_{2}$-Ar Using Intrinsic LowDimensional Manifolds and Wavelet Adaptive Multilevel Representation," Combustion Theory and Modelling, Vol. 5, No. 2, 2001, pp. 163-184.

${ }^{10}$ Williams, F. A., Combustion Theory, Addison-Wesley, Redwood City, 1985, pp. 1-18.
} 
${ }^{11}$ Kee, R. J., Coltrin, M. E., and Glarborg, P., Chemically Reactive Flow: Theory and Practice, John Wiley, Hoboken, NJ., 2003, pp. 649-683.

${ }^{12}$ Golub, G. H., and Van Loan, C. F., Matrix Computations, John Hopkins University, MD., 1983, pp. 251-266.

${ }^{13}$ Miller, J. A., Smooke, M. D., Green, R. M., and Kee, R. J., "Kinetic Modeling of The Oxidation of Ammonia in Flames," Combustion Science and Technology, Vol. 34, Nos. 1-6, 1983, pp. 149-176.

${ }^{14}$ Kee, R. J., and Miller, J. A., "Computational Modeling of Flame Structure," Physica D Vol. 12, Nos. 1-3, 1984, pp. 198211.

${ }^{15}$ Aris, R., Vectors, Tensors, and the Basic Equations of Fluid Mechanics, Dover, NY., 1989, pp. 245-253.

${ }^{16}$ Kee, R. J., Grcar, J. F., Smooke, M. D., and Miller, J. A., "A Fortran Program for Modeling Steady Laminar One Dimensional Premixed Flames," Sandia National Labs., Rept. SAND85-8240, Livermore, CA., 1992.

${ }^{17}$ Kee, R. J., Rupley, F. M., and Miller, J. A., "Chemkin-II: A Fortran Chemical Kinetics Package for the Analysis of Gas Phase Chemical Kinetics," Sandia National Labs., Rept. SAND89-8009B, Livermore, CA., 1992.

${ }^{18}$ Kee, R. J., Rupley, F. M., and Miller, J. A., "The Chemkin Thermodynamic Data Base," Sandia National Labs., Rept. SAND87-8215B, Livermore, CA., 1992.

${ }^{19}$ Kee, R. J., Dixon-Lewis, G., Warnatz, J., Coltrin, M. E., and Miller, J. A., "A Fortran Computer Code Package for the Evaluation of Gas-Phase Multicomponent Transport Properties," Sandia National Labs., Rept. SAND86-8246, Livermore, CA., 1991.

${ }^{20}$ Dixon-Lewis, G., "Kinetic Mechanism, Structure and Properties of Premixed Flames in Hydrogen-Oxygen-Nitrogen Mixtures," Philosophical Transactions of the Royal Society of London. Series A, Vol. 292, No. 1388, 1979, pp. 45-99.

${ }^{21}$ Vincenti, W. G., and Kruger, C. H., Introduction to Physical Gas Dynamics, John Wiley, NY., 1965, pp. 14-26.

${ }^{22}$ Katta, V. R., and Roquemore, W. M., "Numerical Studies on The Structure of Two-Dimensional $\mathrm{H}_{2} /$ Air Premixed Jet Flame," Combustion and Flame, Vol. 102, Nos. 1-2, 1995, pp. 21-40.

${ }^{23}$ Westbrook, C. K., "Hydrogen Oxidation-Kinetics in Gaseous Detonations," Combustion Science and Technology, Vol. 29, Nos. 1-2, 1982, pp. 67-81.

${ }^{24}$ Thiele, M., Warnatz, J., Dreizler, A., Lindenmaier, S., Schießl, R., Maas, U., Grant, A., and Ewart, P., "Spark Ignited Hydrogen/Air Mixtures: Two Dimensional Detailed Modeling and Laser Based Diagnostics," Combustion and Flame, Vol. 128, Nos. 1-2, 2002, pp. 74-87.

${ }^{25}$ Warnatz, J., Maas, U., and Dibble, R. W., Combustion, Springer Verlag, Berlin, 1999, pp. 65-84.

${ }^{26}$ Patnaik, G., and Kailasanath, K., "Numerical Simulations of Burner-Stabilized Hydrogen-Air Flames in Microgravity," Combustion and Flame, Vol. 99, No. 2, 1994, pp. 247-253.

${ }^{27}$ Burks, T. L., and Oran, E. S., " A Computational Study of The Chemical Kinetics of Hydrogen Combustion," Naval Research Lab., NRL Memorandum Rept. 4446, Washington, D.C., 1981.

${ }^{28}$ Baulch, D. L., Drysdale, D. D., Horne, D. G., and Lloyd, A. C., Evaluated Data for High Temperature Reactions, Butterworth, London, 1972.

${ }^{29}$ Hampson, R. F., Braun, W., Brown, R. L., Garvin, D., Herron, J. T., Huie, R. E., Kurylo, M. J., Laufer, A. H., McKinley, J. D., Okabe, H., Scheer, M. D., Tsang, W., and Stedman, D. H., "Survey of Photochemical and Rate Data for Twenty-Eight Reaction of Interest in Atmospheric Chemistry," Journal of Physical and Chemical Reference Data, Vol. 2, No. 2, 1973, pp. 268-312.

${ }^{30}$ Chen, J. H., Hawkes, E. R., Sankaran, R., Mason, S. D., and Im, H. G., "Direct Numerical Simulation of Ignition Front Propagation in a Constant Volume with Temperature Inhomogeneities. I. Fundamental Analysis and Diagnostics," Combustion and Flame, Vol. 145, Nos. 1-2, 2006, pp. 128-144.

Table 1. Comparison of length scales among various models that use detailed kinetics to describe a laminar premixed hydrogen-air flame.

\begin{tabular}{||l|c|c|c|c|c|c|c|c|}
\hline \hline Ref. & Mixture molar ratio & $T_{o},(\mathrm{~K})$ & $p_{o},(\mathrm{~atm})$ & $\ell_{\text {reaction }},(\mathrm{cm})$ & $\ell_{\text {mfp }},(\mathrm{cm})$ & $\ell_{\text {finest }},(\mathrm{cm})$ & $\Delta x,(\mathrm{~cm})$ \\
\hline \hline 22 & $1.26 \mathrm{H}_{2}+\mathrm{O}_{2}+3.76 N_{2}$ & $4.00 \times 10^{2}$ & $1.000 \times 10^{0}$ & $1.52 \times 10^{1}$ & $4.33 \times 10^{-5}$ & $8.05 \times 10^{-4}$ & $2.50 \times 10^{-2}$ \\
24 & $1.19 \mathrm{H}_{2}+\mathrm{O}_{2}+3.76 N_{2}$ & $3.05 \times 10^{2}$ & $0.987 \times 10^{0}$ & $1.31 \times 10^{1}$ & $3.99 \times 10^{-5}$ & $7.56 \times 10^{-4}$ & - \\
26 & $0.59 \mathrm{H}_{2}+\mathrm{O}_{2}+3.76 N_{2}$ & $3.50 \times 10^{2}$ & $1.000 \times 10^{0}$ & $1.49 \times 10^{1}$ & $7.84 \times 10^{-6}$ & $4.35 \times 10^{-5}$ & $3.54 \times 10^{-2}$ \\
\hline \hline
\end{tabular}



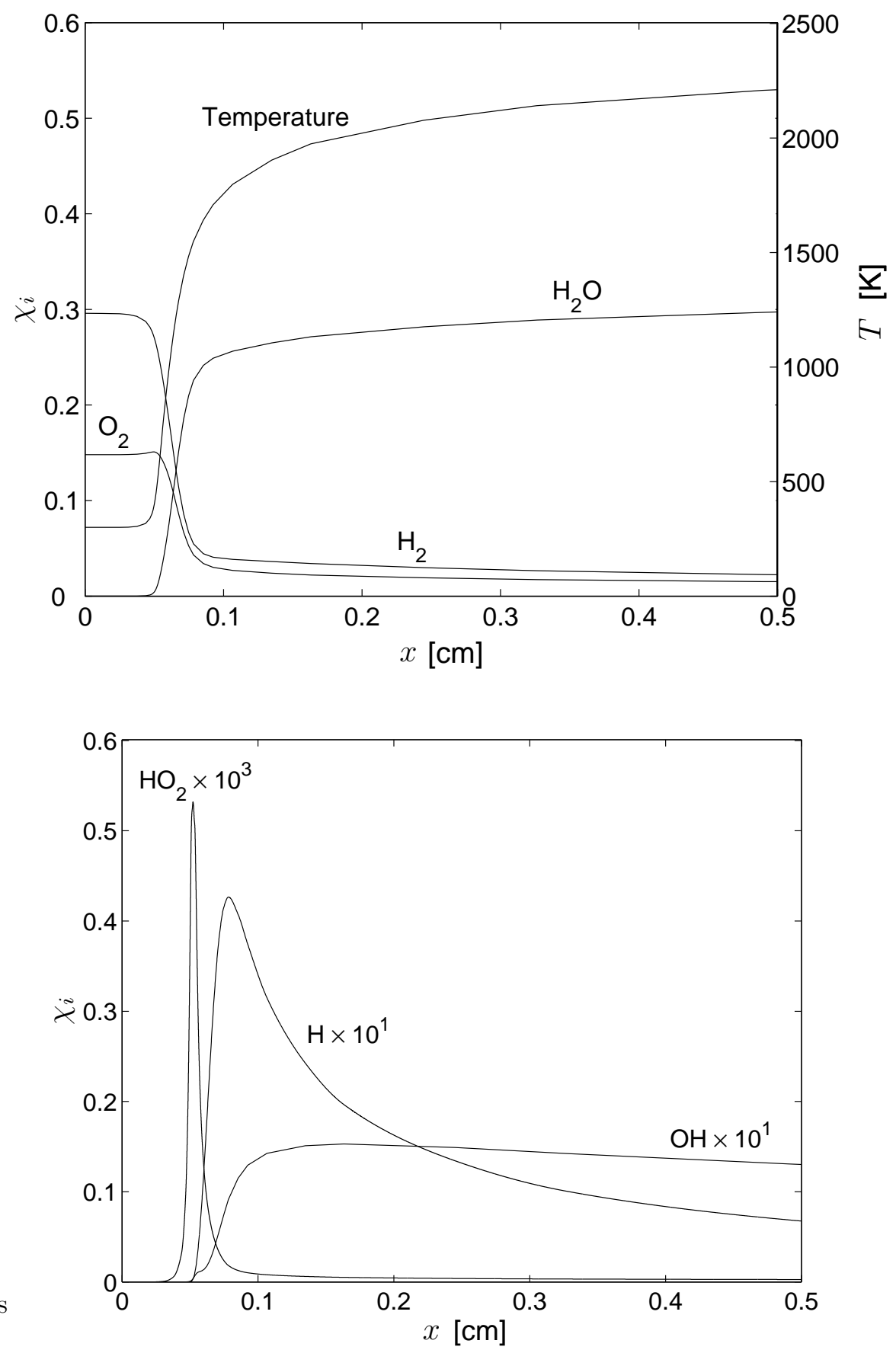

Figure 1. Temperature and species profiles vs. distance in a stoichiometric hydrogen-air flame for numerical verification, equivalent to predictions of Smooke et al., ${ }^{4} T_{o}=298 \mathrm{~K}, p_{o}=1 \mathrm{~atm}$. 


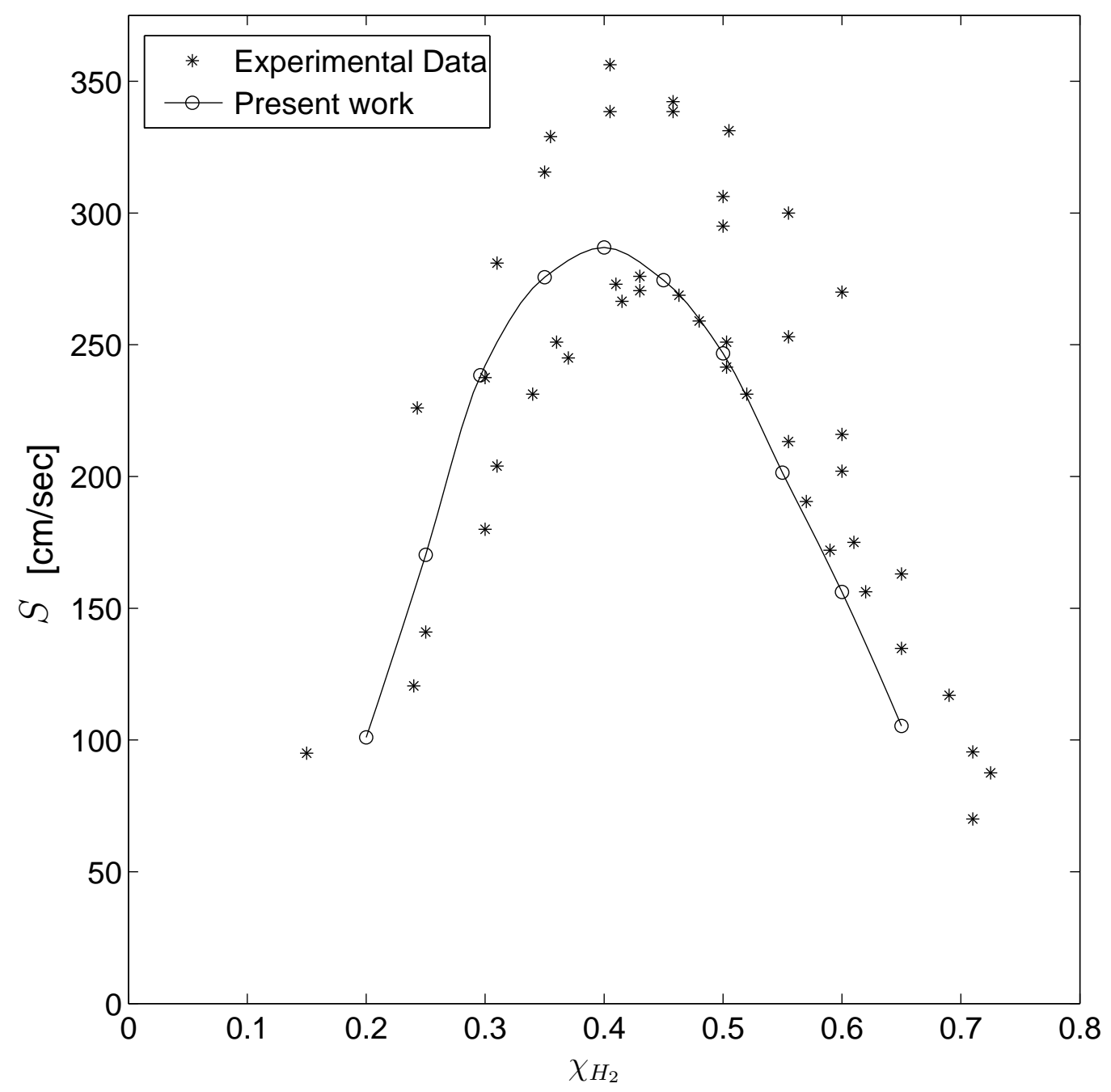

Figure 2. Comparison of predictions of flame speed vs. the unburned diatomic hydrogen mole fraction with the experimental data compiled by Dixon-Lewis, ${ }^{20} T_{o}=298 \mathrm{~K}, p_{o}=1 \mathrm{~atm}$. 


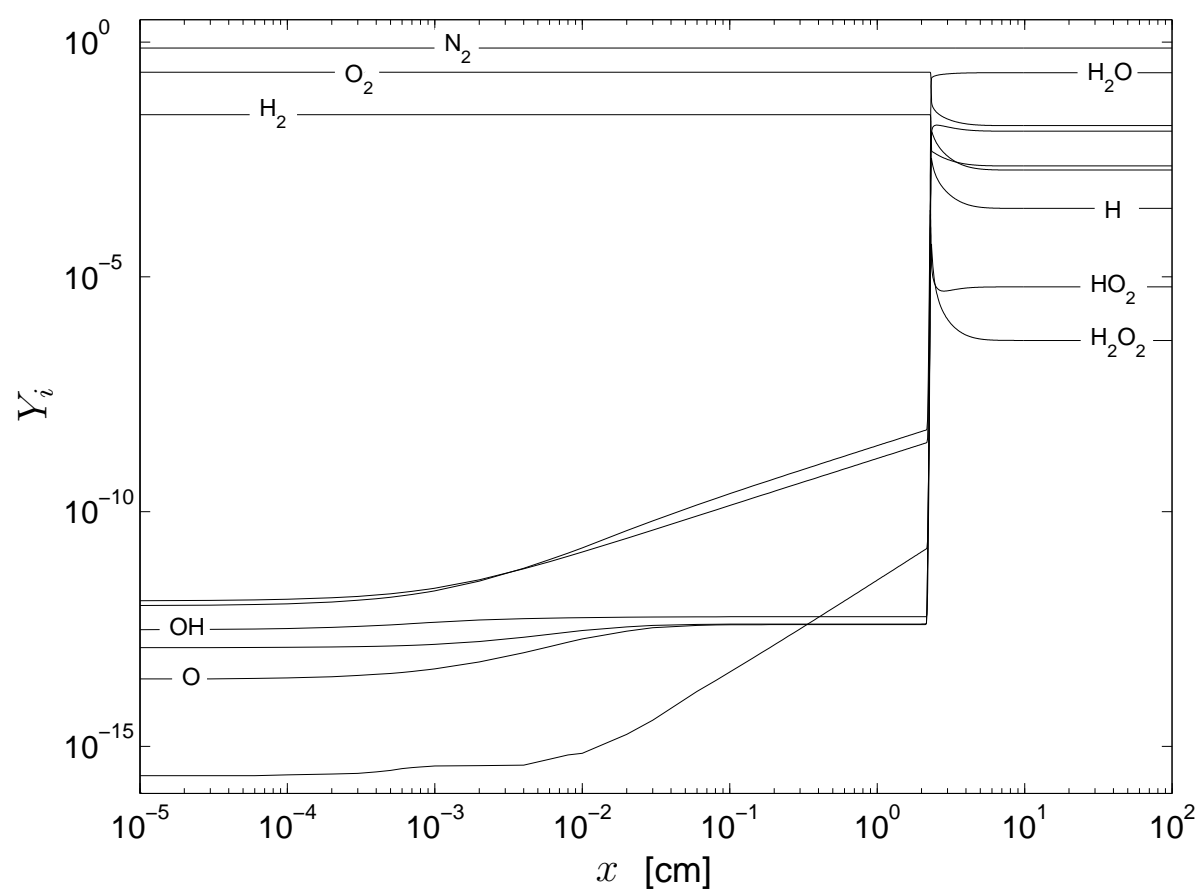

Figure 3. Species mass fraction vs. distance for a stoichiometric hydrogen-air flame, $T_{o}=800 \mathrm{~K}, p_{o}=1 \mathrm{~atm}$.

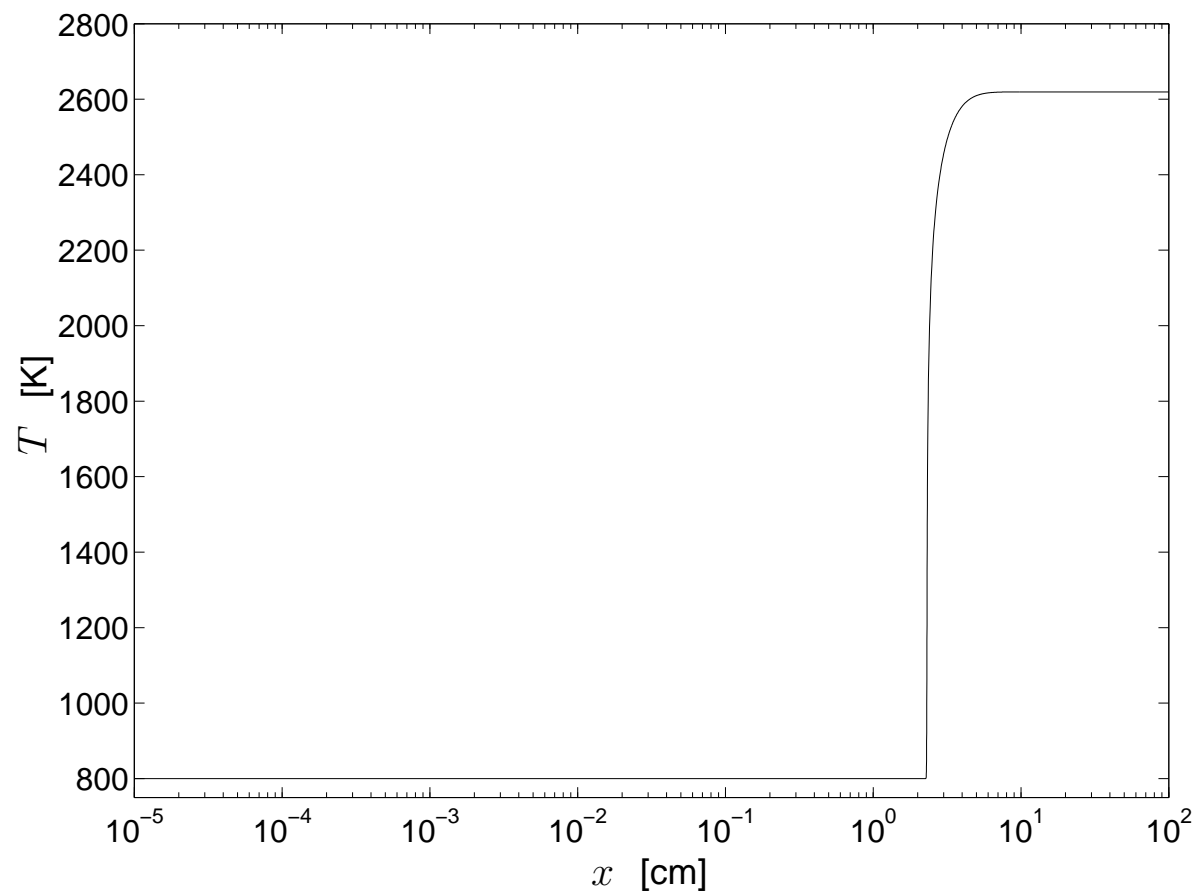

Figure 4. Temperature vs. distance for the stoichiometric hydrogen-air flame, $T_{o}=800 \mathrm{~K}, p_{o}=1 \mathrm{~atm}$. 


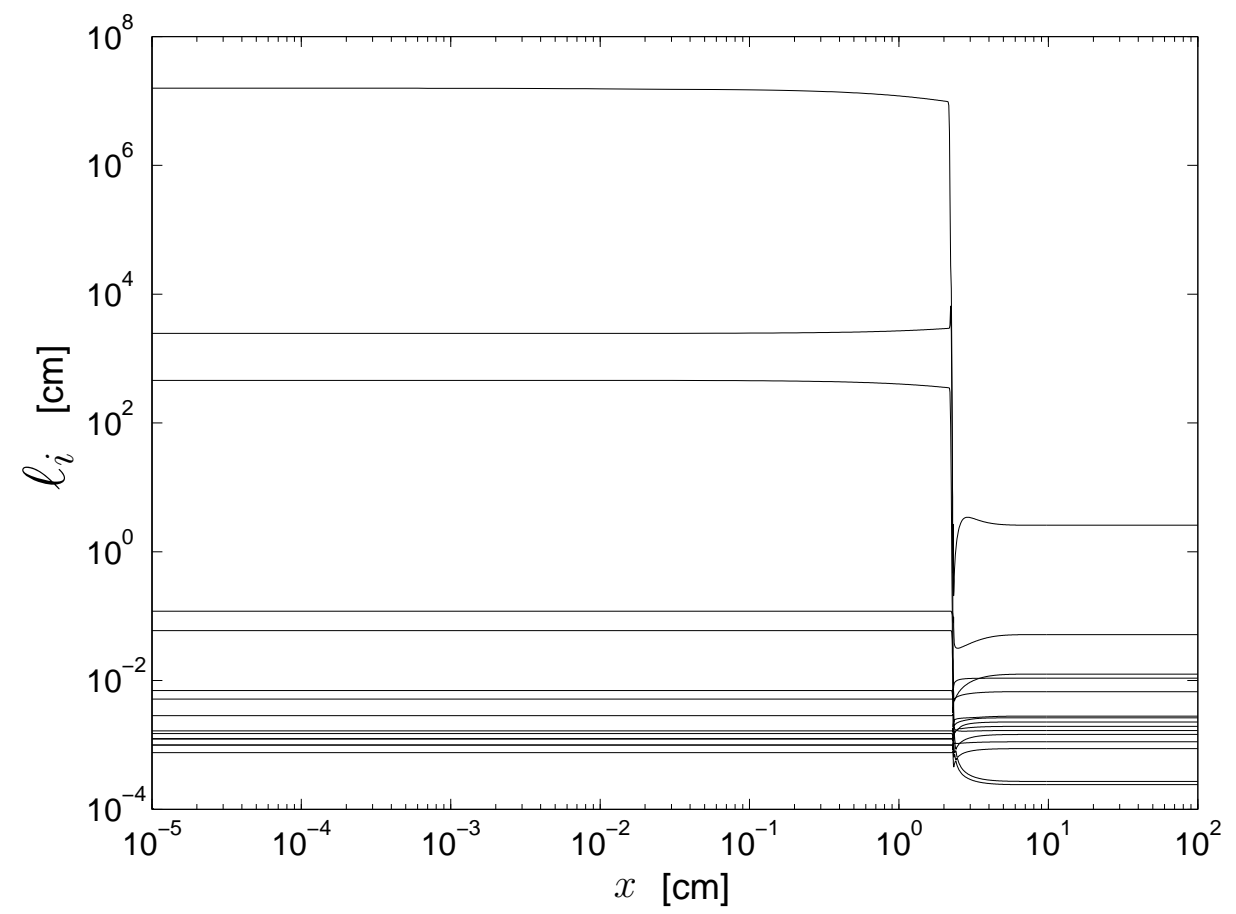

Figure 5. Predicted length scales over which stoichiometric hydrogen-air flame evolve vs. distance, $T_{o}=800 \mathrm{~K}, p_{o}=1 \mathrm{~atm}$.

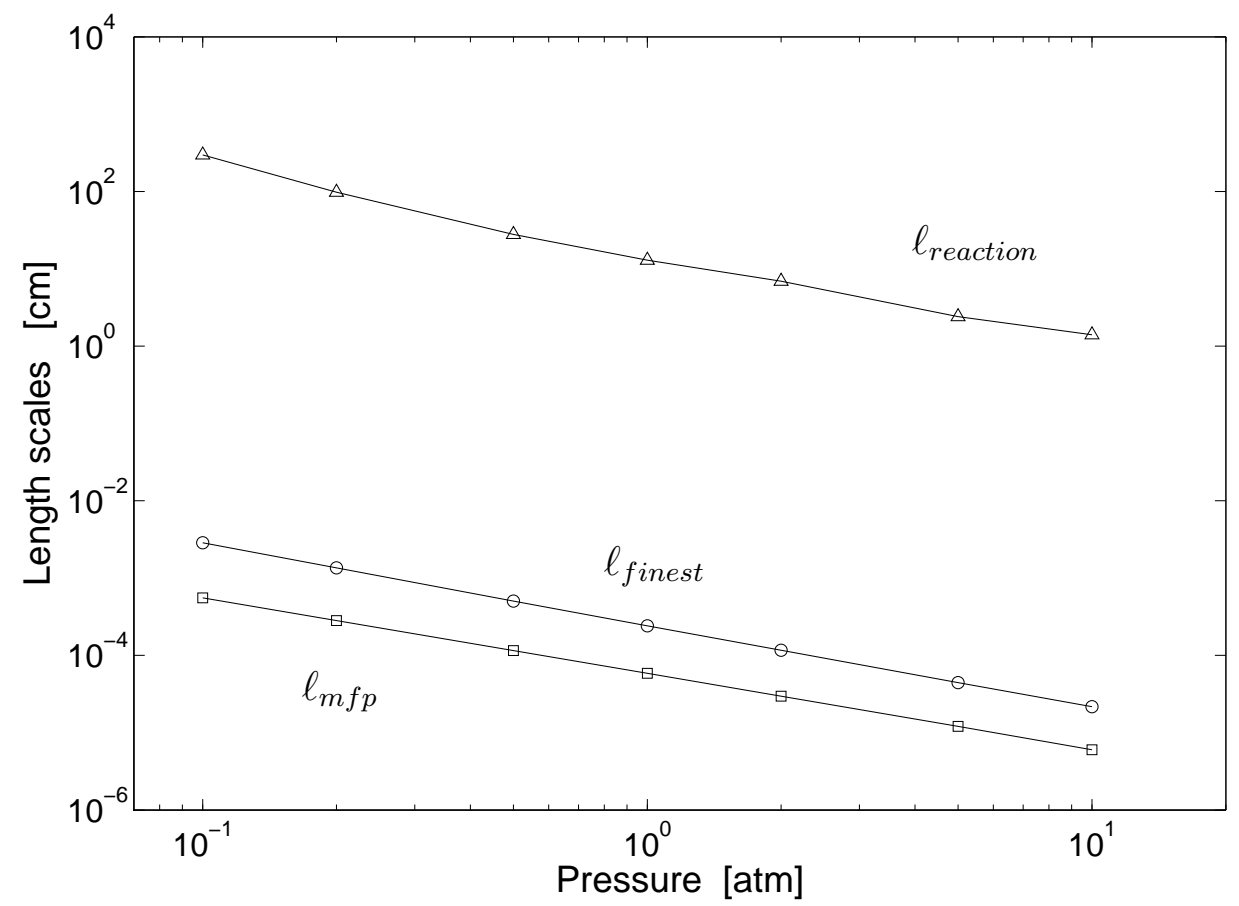

Figure 6. The reaction zone length, the finest length scale predicted by eigenvalue analysis, and the mean free path vs. pressure for stoichiometric hydrogen-air flame, $T_{o}=800 \mathrm{~K}$. 